\title{
O USO DA INTERNET DAS COISAS (IOT) A FAVOR DA SAÚDE
}

\author{
THE USE OF INTERNET OF THINGS (IOT) IN FAVOR OF HEALTH
}

\author{
Silze Cristina Massola - silzemassola@bol.com.br \\ Giuliano Scombatti Pinto - giuliano.pinto@fatectq.edu.br \\ Faculdade de Tecnologia de Taquaritinga (FATEC) - SP - Brasil \\ DOI: 10.31510/infa.v15i2.515
}

\begin{abstract}
RESUMO
Neste trabalho, por meio da introdução da tecnologia da Internet das Coisas, é proposto um novo conceito como aliada na saúde. Ao lidar com o gargalo e o desafio encontrados pelas informações médicas e de saúde, analisa-se que a Internet das Coisas tem grandes vantagens na percepção, transmissão e aplicação de informações, e terá uma ampla perspectiva de aplicação no campo da medicina e nos cuidados da saúde. Com o forte apoio e garantia da tecnologia da Internet das Coisas, uma espécie de sistema inteligente, acessível e comunicativo será a tendência inevitável do desenvolvimento futuro. Este artigo aborda pesquisas, conceitos e discussões sobre a Internet das Coisas no campo da saúde, apresentando sua definição, surgimento, algumas de suas aplicações, os problemas de saúde que surgem com o envelhecimento da população, a tecnologia como revolucionária, a interconexão de dispositivos no mundo, monitoramento remoto, e exemplos concretos de seu uso. O objetivo deste estudo é evidenciar de forma conceitual, a importância da utilização da IoT na área da saúde. E ao final, com as devidas análises e pesquisas, foi possível concluir que, apesar das possíveis barreiras quanto à adoção da IoT, pode-se resultar em muitos benefícios com o seu uso a favor da saúde.
\end{abstract}

Palavras-chave: Futuro. Internet das Coisas. Prevenção. Saúde.

\begin{abstract}
In this work, through the introduction of the Internet technology of Things, a new concept is proposed referring to the same as an ally in health. In dealing with the bottleneck and challenge encountered by medical and health information, it is analyzed that the Internet of Things has great advantages in the perception, transmission and application of information, and will have a wide perspective of application in the field of medicine and in the care of health. With the strong support and assurance of Internet technology of Things, a kind of intelligent, accessible and communicative system will be the inevitable trend of future development. This article addresses research, concepts and discussions on the internet of things in the health field, presenting its definition, emergence, some of its applications, health problems that arise with the aging population, technology as revolutionary, the interconnection of devices in the world, remote monitoring, and concrete examples of its use. The objective of this study is to demonstrate in a conceptual way, the importance of the use of IoT in the health area. And finally, with the appropriate analysis and research, it was possible to conclude that, despite the possible barriers to the adoption of IoT, it can result in many benefits from its use in favor of health.
\end{abstract}


Keywords: Future. Internet of Things. Prevention. Health.

\title{
1 INTRODUÇÃO
}

A saúde é um dos fatores mais importantes para o ser humano. De acordo com o Artigo 196 da Constituição do Brasil (BRASIL, 1988) "a saúde é direito de todos e dever do Estado, garantindo mediante políticas sociais e econômicas que visem à redução do risco de doença e de outros agravos e ao acesso universal e igualitário às ações e serviços para sua promoção, proteção e recuperação."

O texto da Constituição da República Federativa do Brasil (BRASIL, 1988), em seu artigo 198 sintetiza o seguinte:

\begin{abstract}
As ações e serviços públicos de saúde integram uma rede regionalizada e hierarquizada e constituem um sistema único, organizado de acordo com as seguintes diretrizes: descentralização, com direção única em cada esfera de governo; atendimento integral, com prioridade para as atividades preventivas, sem prejuízo dos serviços assistenciais e participação da comunidade. (BRASIL, 1988).
\end{abstract}

Diante disso, todos devem ter acesso irrestrito à saúde com atendimento multidisciplinar e integral, de maneira equitativa, com participação comunitária nas decisões e com uma gestão hierarquizada de autonomia regional.

De acordo com dados do Instituto Brasileiro de Geografia e Estatística (IBGE, 2015), as doenças crônicas não transmissíveis (DCNT) constituíram o problema de saúde de maior magnitude relevante, e responderam por mais de $70 \%$ das causas de mortes no Brasil. As doenças cardiovasculares, câncer, diabetes, enfermidades respiratórias crônicas e doenças neuropsiquiátricas, principais DCNT, responderam por um número elevado de mortes antes dos 70 anos de idade e perda de qualidade de vida, gerando incapacidades e alto grau de limitação das pessoas doentes em suas atividades de trabalho e de lazer. Segundo Gaziano, Galea e Reddy (2007), citados em publicação de pesquisa do IBGE (2014), há uma forte associação das principais DCNT a fatores de riscos altamente prevalentes, destacando-se o tabagismo, consumo abusivo de álcool, excesso de peso, níveis elevados de colesterol, baixo consumo de frutas e verduras e sedentarismo. O monitoramento destes fatores de risco e da prevalência das doenças a eles relacionados é primordial para definição de políticas de saúde voltadas para prevenção destes agravos. 
De acordo com a publicação da Vertical Saúde ACATE (2017), a medida em que emergiram estudos relacionando fatores causais no processo de saúde-doença, a medicina preventiva começou a expressar-se como grande promissora no campo da saúde. Afinal, não existe solução melhor do que prevenir o aparecimento da doença, dos seus custos e de suas comorbidades associadas.

Hipócrates- o pai da medicina- já diria: "Primum non nocere". Em tradução: “primeiro, não causar dano" (NORMAN, 2009).

A medicina preventiva vem com muitas promessas para o futuro, mas cabe aos profissionais de saúde serem resistentes tanto à pressão da corporação farmacêutica, tecnológica e profissional, como também dos pacientes.

Já no artigo 218 (caput, par. $1^{\circ}$ e par. $2^{\circ}$ ), a Constituição trata do seguinte:

O Estado promoverá e incentivará o desenvolvimento científico, a pesquisa, a capacitação científica e tecnológica e a inovação. A pesquisa científica básica e tecnológica receberá tratamento prioritário do Estado, tendo em vista o bem público e o progresso da ciência, tecnologia e inovação. A pesquisa tecnológica voltar-se-á preponderantemente para a solução dos problemas brasileiros e para o desenvolvimento do sistema produtivo nacional e regional. (BRASIL, 1988).

Diante do que é assegurado pela constituição para todo cidadão, como o direito à saúde, com prioridade para a prevenção, e a tecnologia visando a solução dos problemas brasileiros, um fator interessante é interligar as duas coisas, trazendo benefícios na área da saúde, como por exemplo, aparelhos que fazem leitura de pressão sanguínea e monitoramento cardíaco, podendo estar conectados com hospitais, clínicas médicas ou empresas de plano de saúde.

Dentro deste contexto, entra a Internet das Coisas, aliada aos conhecimentos médicos como forma de atuar principalmente na medicina preventiva, promovendo melhor qualidade de vida e bem-estar aos pacientes, menos custos com medicamentos, consultas e demais procedimentos médicos. IoT (Internet of Things), Internet das Coisas em português, foi mencionada pela primeira vez em 1999, por Kevin Ashton numa apresentação para executivos da Procter \& Gamble. "É um sistema de computação de dispositivos inter-relacionados, mecânicos e digitais, objetos, animais ou pessoas equipadas com identificadores únicos e com a habilidade de transferir dados através de uma rede sem requerer a interação entre humanos ou humano e computador" (COSTA, 2018).

A Internet das Coisas pode contribuir com os profissionais da saúde, com hospitais e centros de saúde, podendo estes, a concentrarem seus esforços e recursos em casos mais sérios e inevitáveis, como o combate ao câncer, por exemplo. 


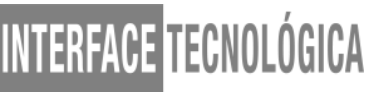

O objetivo deste trabalho é mostrar de forma conceitual, através de pesquisas bibliográficas, que a tecnologia da Internet das Coisas está chegando para revolucionar o mercado da saúde, não só para pacientes, mas para a sociedade em geral.

Visando verificar as vantagens do uso das tecnologias da Internet das Coisas, algumas de suas desvantagens, suas principais aplicações na área da saúde e precauções, para o presente estudo utilizou-se como metodologia uma revisão bibliográfica, através de pesquisas científicas em livros, artigos, revistas, jornais, legislações, estatísticas e demais fontes referenciadas neste artigo.

\section{FUNDAMENTAÇÃO TEORICA}

\subsection{Internet das Coisas}

A Internet das Coisas pode ser definida como "um novo mundo em que os objetos estarão conectados e passarão a realizar tarefas sem a interferência humana" (ASHTON ,2015).

IOT pode ser contemplada como uma estrutura de rede abrangente, consistindo de vários tipos de objetos, que dependem de tecnologias de sensores, de comunicação, de rede e de processamento de informações. A tecnologia base para IOT é identificadores de rádio frequência (RFID, em inglês), que permite que os microchips transfiram dados de identificação para o leitor por meio sem fio. Através dessa tecnologia, pode-se analisar, rastrear e monitorar os objetos conectados com suas tags. Outra tecnologia fundamental é a Wireless Sensor Networks (WSNs), que funciona principalmente em sensores inteligentes para detecção e monitoramento. A RFID encontra sua aplicação no transporte de mercadorias aos consumidores, produção de produtos farmacêuticos e varejo desde o ano 1980 e a WSN se aplica ao tráfego, saúde e monitoramento. O avanço em ambas as tecnologias acelera o crescimento da IoT. Muitas outras tecnologias e dispositivos, incluindo códigos de barras, serviços baseados em localização, SoA, comunicação de campo próximo, Wimax, ZigBee, computação em nuvem, etc. também já estão começando a fazer uma rede abrangente para fortalecer a IoT (MEHTA, 2018).

A ideia básica do IoT será permitir uma conexão autônoma e segura e troca de dados entre dispositivos e aplicações do mundo real (Fan e Chen, 2010) onde o IoT vai incorporar 
alguma inteligência em objetos conectados à Internet para se comunicar, trocar informações, tomar decisões, invocar ações. Tende assim fornecer serviços surpreendentes que o torna em crescente popularidade para as instituições acadêmicas, indústrias, bem como governos uma vez que tem o potencial de trazer significativos benefícios pessoais, profissionais e econômicos.

É a computação facilitando a vida, deixando as pessoas livre para fazer outras coisas.

\subsection{Surgimento da IoT}

Conforme Ashton (2015), o termo IoT foi originado em 1999, quando ele escreveu um artigo chamado "As coisas da Internet das Coisas". Segundo ele, a falta de tempo das pessoas abre portas para que ferramentas sejam criadas para fazer coisas que, de fato, não necessitam ser feitas por pessoas. Podem ser substituídas por dispositivos. Dispositivos esses, que conversando por diferentes protocolos dentro da mesma rede, conseguem acompanhar as pessoas, ler suas atividades, gerar informações e a partir daí auxiliá-las no dia a dia.

"Nossa economia, sociedade e sobrevivência não são baseadas em ideias ou informações - elas são baseadas em coisas. Ideias e informações são importantes, mas as coisas importam muito mais", disse Ashton em sua entrevista ao jornal RFID (2009).

Através de RFID, e tecnologia de sensores, os computadores poderão observar, identificar e entender o mundo, sem as limitações de dados inseridos pelo ser humano.

\subsection{Algumas aplicações da IoT}

A Internet das Coisas pode ser usada para uma ampla variedade de aplicações, incluindo residências, sistemas de energia, segurança e vigilância, transporte, agricultura, saúde, indústria, logística e meio ambiente, como pode ser observado na Figura 1. 
Figura 1- Exemplos de algumas aplicações IoT

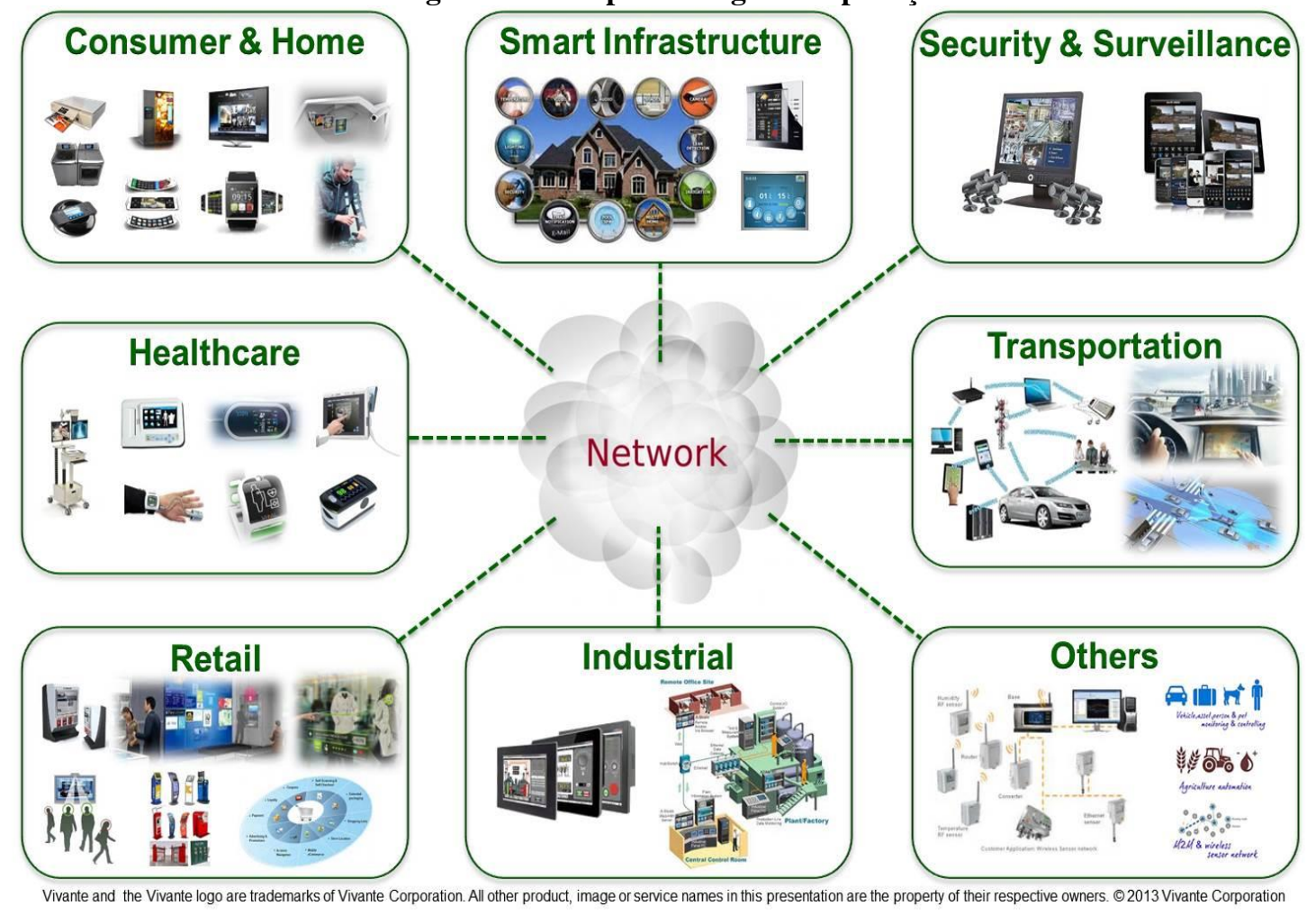

Fonte: Vivante Corporation (2013)

São muitos os domínios de aplicações que serão impactados pela IoT. Podem ser classificadas com base no tipo de disponibilidade de rede, de cobertura, heterogeneidade, escala e envolvimento do usuário. Não há quase nenhuma área de aplicação onde o IoT não consiga encontrar uma função e principalmente não há área de aplicação onde a IoT não traga alguma vantagem econômica ao longo do tempo (SILVA, 2017).

\subsection{Um problema global}

Hoje, o envelhecimento global e a prevalência de doenças crônicas tornaram-se uma preocupação comum. Muitos países estão passando por uma reestruturação hospitalar, reduzindo número de leitos hospitalares e aumentando a proporção de cuidados de saúde. Uma tendência promissora na saúde é mudar exames médicos de rotina e outros serviços de saúde de hospital para o ambiente doméstico. Para que os pacientes, assim, possam obter cuidados de saúde sem intervenções dentro de um ambiente doméstico confortável, liberando recursos hospitalares para pessoas que precisam de cuidados de emergência. Para acompanhar 


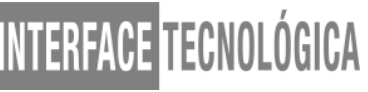

o estado físico dos idosos e mantê-los saudáveis, duas tarefas diárias são essenciais: monitoramento em tempo real e análise de sinais vitais para detectar ou prever precocemente eventos adversos com risco de vida. Um estudo publicado no jornal de pesquisa psicossomática em 2011, estima que cerca de 25\% da população adulta não aderem à sua medicação prescrita, o que pode levar a desfechos de saúde e aumento da mortalidade. Baixa adesão à medicação é um grande problema para os indivíduos e prestadores de cuidados de saúde. Melhorias tecnológicas em instalações e serviços de saúde são altamente desejáveis para atender as exigências deste grupo gigante (YANG et al., 2014).

\subsection{Um mundo de dispositivos conectados}

A Internet das Coisas não é mais uma promessa tecnológica. Os dispositivos conectados estarão em todos os lugares. Dentro de casas e carros, na estrutura física das cidades, no processo produtivo industrial e agrícola, nas cadeias logísticas de transporte, nos serviços e, inclusive no corpo das pessoas, com o uso dos dispositivos vestíveis, como relógios, sapatos e outras peças de vestuário.

A IoT é a base do processo de digitalização da economia, que tem transformado os métodos tradicionais de produção, no uso das tecnologias de informação e comunicação e na interconexão de dispositivos. A conexão entre sistemas de TI, subsistemas, processos, objetos e aplicativos, que se comunicam entre si e com humanos, é o vetor chave dessa transformação, e é esperado que as aplicações de IoT e as tecnologias digitais promovam ganhos de produtividade e competitividade das nações. Isso ganha ainda maior importância para o Brasil, dado que nas últimas décadas vem perdendo sua capacidade de agregação de valor da produção industrial em comparação às demais economias em desenvolvimento e também perdeu posições no ranking de competitividade industrial para países emergentes, mostrando o quão iminente é que o País tome ações para estimular o desenvolvimento de tecnologias que garantam maior produtividade e competitividade nos setores básicos da economia. É importante também destacar a relevância do envolvimento de setores de governo, empresarial e comunidade científica em iniciativas voltadas à adoção e ao desenvolvimento da Internet das Coisas e das novas tecnologias digitais; à padronização de aplicações digitais; à segurança digital; à modernização do marco legal; à formação e à capacitação profissional; e à melhoria do ambiente de negócios e da infraestrutura. Face a esse 
reconhecimento, esforços têm sido destinados à expansão do uso de IoT e das tecnologias digitais no Brasil. O Ministério da Ciência, Tecnologia, Inovações e Comunicações(MCTIC), juntamente com a iniciativa privada, academia e órgãos de governo num esforço de construção de uma política pública para o tema, conseguiu a criação do Plano Nacional de Internet das Coisas, cujo objetivo é "acelerar a implantação da Internet das Coisas como instrumento de desenvolvimento sustentável da sociedade brasileira, capaz de aumentar a competitividade da economia, fortalecer as cadeias produtivas nacionais e promover a melhoria da qualidade de vida". Todos os agentes envolvidos na criação do Plano compartilham da aspiração de que a Internet das Coisas tenha um impacto positivo, relevante e rápido na economia e na vida das pessoas. Para atingir esse objetivo, o Plano Nacional de IoT atuará em diversas dimensões, estabelecendo diretrizes gerais e propondo iniciativas concretas (ESTRATÉGIA brasileira para a transformação digital, 2018).

Diante destes dados, observa-se a importância da valorização de investimentos em tecnologias, para alavancar o ranking do Brasil nos setores tecnológicos, já que acabou perdendo posição diante de outros países, em anos anteriores.

\subsection{IoT e monitoramento remoto}

O surgimento da Internet das Coisas possibilitou uma série de avanços tecnológicos na saúde. Por meio de dispositivos conectados remotamente é possível reunir automaticamente uma série de informações sobre o estado da saúde do paciente, que ajudam no trabalho de diagnóstico e posterior tratamento ou prevenção.

Conforme a empresa de consultoria Tractica (2016), com a ajuda da IoT é possível medir indicadores como a pressão arterial, batimentos cardíacos e temperatura corporal à distância. Esses dados são enviados para aplicativos e analisados pelos profissionais da saúde. Com isso, é possível evitar uma série de complicações decorrentes da falta de atendimento, tomando as medidas necessárias o mais rapidamente possível e diminuindo consideravelmente os índices de hospitalização desnecessárias.

Também há os chamados wearables, que são dispositivos usados como peças de vestuário, conectados à internet, que transmitem em tempo real atualizações sobre o estado de saúde do paciente. Hoje existem pulseiras, colares ou relógios que enviam automaticamente um sinal para a equipe de saúde caso algum indicador fuja dos parâmetros de controle. De 


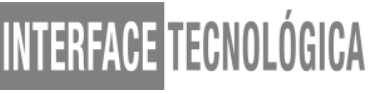

acordo com um relatório da consultoria Tractica (2016), as remessas mundiais de produtos de saúde aumentarão de 2,5 milhões em 2016 para 97,6 milhões de unidades anualmente até 2021. No final desse período, a firma de inteligência de mercado prevê que o mercado global de produtos de saúde será responsável por US \$17,8. bilhões em receita anual,

A tecnologia chegou para ficar e a tendência para os próximos anos é que seu uso se torne cada vez mais comum no universo da saúde.

\section{PROCEDIMENTOS METODOLÓGICOS}

Visando verificar as vantagens do uso das tecnologias da internet das coisas, algumas de suas desvantagens, suas principais aplicações na área da saúde e precauções, para o presente estudo utilizou-se como metodologia uma revisão bibliográfica, através de pesquisas científicas em livros, artigos, revistas, jornais, legislações, estatísticas e demais fontes referenciadas neste artigo.

\section{RESULTADOS E DISCUSSÕES}

São inúmeras as aplicações da IoT na área da saúde, como citado no decorrer deste trabalho, mas para análise de estudo será apresentado alguns casos concretos de seus muitos usos na área da saúde.

\subsection{Uso da IoT em um hospital de Boston para acompanhar os recém-nascidos na UTIN (unidade de terapia intensiva neonatal)}

Neste hospital, sensores são utilizados para fins de segurança. Bebês recém-nascidos recebem pulseiras, permitindo que uma rede sem fio os localize a qualquer momento. Se um recém-nascido for levado muito perto de uma porta de saída sem ser desconectado, os elevadores irão parar e as portas de saída serão travadas. E na unidade de terapia intensiva neonatal, os enfermeiros recebem alertas em telefones celulares hospitalares sobre as condições médicas de seus pacientes, incluindo a frequência cardíaca e as mudanças de oxigênio que os sensores detectaram, permitindo que eles cheguem ao leito dos pacientes mais rapidamente. Além disso, o uso da IoT nos serviços de saúde permitiu que o hospital 
atualizasse prontuários eletrônicos mais rapidamente. O hospital também instalou sensores sem fio em geladeiras, freezers e laboratórios para garantir que amostras de sangue, medicamentos e outros materiais sejam mantidos nas temperaturas adequadas (GUIA ESSENCIAL TECHTARGET, 2016).

Segundo Jim Piepenbrink, diretor de engenharia clínica do hospital de Boston, as temperaturas eram registradas e documentadas manualmente. Mas agora, com a instalação de sensores sem fio, gerou uma grande economia de tempo para a equipe de saúde do local.

\subsection{IoT para gerenciamento de estoque em hospital}

Conforme Ganguly (2016), os hospitais não estão usando a IoT para rastrear estoque da maneira mais ampla que seria desejável. Ele acrescentou que a saúde poderia aprender algumas lições do varejo.

Para os hospitais, a grande vantagem que eles podem obter do gerenciamento de estoques com a IoT será em áreas como farmácia e controle geral de estoque em depósitos.

\subsection{A ajuda do monitoramento de IoT em um paciente com insuficiência cardíaca crônica}

Sem acesso a séries temporais contínuas de dados, os médicos muitas vezes não conseguem detectar mudanças críticas nas condições do paciente com antecedência suficiente para prevenir emergências. Por exemplo, atualmente um paciente que mora no Reino Unido, de meia-idade e um pouco acima do peso, sofre de insuficiência cardíaca crônica, hipertensão arterial e diabetes tipo 2. Ele está sendo tratado com um diurético, seguindo as recomendações de dieta e exercícios de seu médico. Durante as férias, acaba relaxando com suas restrições e sente-se um pouco mais inchado, mas não se preocupando com isso. Algumas semanas antes de seu próximo check-up, ele entra em colapso com insuficiência cardíaca crônica, é hospitalizado por 12 dias a um custo de US \$ 4.500, pago pelo SUS, e em seguida, passa por uma estadia de reabilitação. Futuramente, com o uso da IoT, seria possível que o paciente, com quatro dispositivos conectados, sendo: uma balança de peso, um medidor de pressão arterial, uma caixa de comprimidos inteligente e uma pulseira que rastreie sua frequência cardíaca e nível de oxigênio no sangue (custando menos de US \$ 300), pudessem detectar 
rapidamente uma mudança em sua condição, percebendo que ele está ficando cansado mais rapidamente durante as caminhadas e que não está tomando corretamente seus medicamentos durante as férias. É importante ressaltar que a balança também captou um aumento de $2 \mathrm{~kg}$ em seu peso, em apenas alguns dias - um sinal de aumento da retenção de líquidos. Tudo indica então, que deve ser solicitado uma consulta imediata ao seu médico e talvez um aumento na dosagem de seu diurético. Seu médico recebe um alerta com todas essas informações e lhe agenda uma consulta rapidamente, lembrando o paciente como é importante não faltar ao diurético. Em uma visita na semana seguinte, o médico vê que o peso do paciente caiu e ele é capaz de respirar facilmente durante as atividades normais, recomendando que ele siga a uma dieta rigorosa e tome seus medicamentos fielmente, mesmo durante feriados. Estimativas do Mckinsey Global Institute (2015), mostram que os aplicativos de IoT podem reduzir o custo dos cuidados com doenças crônicas a pacientes em 10 a 15\%, baseados em experiência de dados clínicos recentes. Alguns testes de monitoramento remoto indicaram potenciais reduções de custos superior a 50\% no tratamento de populações agudas, mas espera-se que essa grande redução de custos não seja sustentável em populações maiores de pacientes. No entanto, se a tecnologia de saúde é capaz de atingir todo o seu potencial para melhorar a adesão a terapias prescritas, a IoT poderia reduzir o custo do tratamento de um paciente com doenças em $50 \%$. Benefícios adicionais podem ser obtidos se os sistemas baseados em IoT puderem gerar mudanças substanciais em dieta e exercício. Atualmente, a capacidade de incentivar essas mudanças no estilo de vida é limitada. Com dados de monitoramento baseados em IoT, há mais oportunidades de feedback e reforço de prestadores de cuidados de saúde, de outros pacientes e familiares (MCKINSEY Global Institute, 2015).

A Internet das Coisas poderia possibilitar incentivos financeiros para pacientes que demonstrem comportamentos de estilo de vida mais saudáveis.

\section{CONSIDERAÇÕES FINAIS}

Embora existam muitos benefícios da Internet das coisas na área da saúde, não são poucos os seus desafios. Como acontece com qualquer nova tecnologia na área da saúde, os executivos da área ficam preocupados com a segurança dos dados e com o gerenciamento de dispositivos da IoT. 


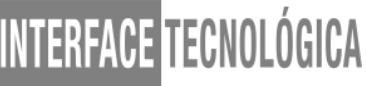

Com ações governamentais para incentivar a interoperabilidade, garantir a segurança e a privacidade dos dados e dos indivíduos, os direitos de propriedade, a internet das coisas pode começar a atingir seu potencial máximo, especialmente se os líderes realmente adotarem a tomada de decisões baseada em dados.

A tecnologia tem sido uma ferramenta valiosa para os médicos, por exemplo, permitindo-lhes gerenciar os dados dos pacientes, acessá-los a partir de seus dispositivos móveis e compartilhá-los com as equipes de atendimento.

O futuro da IoT na área da saúde pode depender da resolução dessas preocupações e da maximização dos benefícios centrais da IoT, como rapidez, transparência e preço.

\section{REFERÊNCIAS}

ASHTON, Kevin. That 'Internet of Things' Thing. RFID Journal.2009. Disponível em: https://www.rfidjournal.com/articles/view?4986.Acesso em: 02 out. 2018.

Entrevista exclusiva com o criador do termo "Internet das Coisas". 2015. Finep. Disponível em: http://finep.gov.br/noticias/todas-noticias/4446-kevin-ashton-entrevistaexclusiva-com-o-criador-do-termo-internet-das-coisas. Acesso em: 02 out. 2018.

AVANÇOS tecnológicos na saúde: quais soluções aplicar.2016. Portal Telemedicina. Disponível em: http://portaltelemedicina.com.br/avancos-tecnologicos-na-saude-aplicacoes/. Acesso em: 26 set. 2018.

BRASIL. Constituição da República Federativa do Brasil de 1988. Brasília, 1988. Disponível em: http://www.planalto.gov.br/ccivil_03/Constituicao/Constituicao.htm. Acesso em: 22 set. 2018.

COSTA, Pedro Miguel Borges da Palma. Sistema de Internet das Coisas para o Apoio aos Cuidados de Saúde da População Idosa. Dissertação de Mestrado do Curso de Engenharia Informática, da Universidade de Évora Escola de Ciências e Tecnologia. 2018. 87 páginas. Disponível em: http://hdl.handle.net/10174/23209. Acesso em: 23 set. 2018.

CUIDADOS de Saúde Primários-Agora mais que nunca. Relatório mundial de Saúde 2008. Disponível em: http://www.who.int/eportuguese/publications/whr08_pr.pdf.Acesso em: 23 set. 2018.

DIAGNÓSTICO Brasil: Principais Problemas da Saúde. Vertical Saúde Acate. 2017. Disponível em: http://saude.acate.com.br/diagnostico-brasil-principais-problemas-da-saude/. Acesso em: 22 set.2018.

ESTRATÉGIA brasileira para a transformação digital. Brasília, 2018. Disponível em: http://www.mctic.gov.br/mctic/opencms/publicacao/publicacoes.html-estrategia_digital.pdf. 
Acesso em: 02 out. 2018.

ELECTRONICS HUB. Internet of Things.2015. Disponível em:

https://www.electronicshub.org/internet-of-things/\#Medical_and_Healthcare_Systems.

INTERNET of Things in healthcare keeps patients healthy, safe.2016.A guide to healthcare IoT possibilities and obstacles.Disponível em:

https://internetofthingsagenda.techtarget.com/feature/Internet-of-Things-in-healthcare-keepspatients-healthy-safe. Acesso em: 03 out. 2018.

IBGE. Pesquisa Nacional de Saúde 2013. Rio de Janeiro, 2014.Disponível em: https://biblioteca.ibge.gov.br/visualizacao/livros/liv91110.pdf.Acesso em: 29 set. 2018.

MCKINSEY Global Institute. Unlocking the Potential of the Internet of Things. 2015.

Disponível em: https://www.mckinsey.com/business-functions/digital-mckinsey/ourinsights/the-internet-of-things-the-value-of-digitizing-the-physical-world. Acesso em: 02 out. 2018.

MEDICINA preventiva/preditiva - qual o futuro usando tecnologia. Vertical Saúde Acate. 2017. Disponível em: http://saude.acate.com.br/medicina-preventiva-preditiva-qual-o-futurousando-tecnologia/.Acesso em: 22 set. 2018.

MEHTAA Rishika, Sahnib Jyoti, Khannac Kavita. International Conference on Computational Intelligence and Data Science.Internet of Things: Vision, Applications and Challenges. 2018. Disponível em:

https://www.sciencedirect.com/science/article/pii/S1877050918307749. Acesso em: 05 out. 2018.

NORMAN, A.H.; TESSER, C. D. Prevenção quaternária na atenção primária à saúde: uma necessidade do Sistema Único de Saúde. Florianópolis.2009. Disponível em: https://www.scielosp.org/article/csp/2009.v25n9/2012-2020/. Acesso em: 05 out.2018.

PORTAL telemedicina. Entenda de uma vez por todas o que é internet das coisas!.2016. Disponível em: http://portaltelemedicina.com.br/internet-das-coisas/. Acesso em: 22 set. 2018.

REMESSAS de Dispositivos Vestíveis de Assistência Médica para Chegar a 98 Milhões de Unidades Anualmente até 2021. 2016. Tractica. Disponível em:

https://www.tractica.com/newsroom/press-releases/healthcare-wearable-device-shipments-toreach-98-million-units-annually-by-2021.Acesso em: 26 set. 2018.

SILVA, Leandro Jamir. Universidade do Sul de Santa Catarina [UNISUL]. Monografia de Trabalho de Conclusão de Curso. Internet Das Coisas. Disponível em: https://riuni.unisul.br/handle/12345/3940. Acesso em: 05 out. 2018.

TECHTARGET. Essential Guide. IOT for healthcare: Three use cases. 2016. Disponível em: https://internetofthingsagenda.techtarget.com/feature/IoT-for-healthcare-Three-use-cases. Acesso em: 29 set. 2018. 


\section{INTERFAGETECENOLOGGCA}

YANG, G. et al. A Health-IoT Platform Based on the Integration of Intelligent Packaging, Unobtrusive Bio-Sensor and Intelligent Medicine Box. IEEE Transactions on Industrial Informatics. Disponível em: http://www.divaportal.org/smash/get/diva2:833968/FULLTEXT01.pdf.Acesso em: 23 set. 2018. 